\title{
Multi-modal SEM Imaging for Shale Reservoir Characterization
}

\author{
Lori A. Hathon ${ }^{1}$, Michael T. Myers ${ }^{1}$, Mike Dixon ${ }^{2}$ and Kultaransingh Hooghan ${ }^{2}$ \\ 1. Department of Petroleum Engineering, University of Houston \\ 2. Weatherford International
}

Recent advances in imaging technologies have allowed us to interrogate materials at progressively higher resolutions over larger areas or volumes of sample. As we have begun to investigate geomaterials at resolutions on the single nanometer scale, our observations have upended several long-lived paradigms of geoscience. This has proved particularly true in shale reservoirs. Although it is fascinating to continue to look more deeply into these reservoirs, we are also faced with three daunting challenges. The first involves development of standard protocols for imaging. As we push the limits of our imaging tools, the details of sample preparation, the composition and thickness of conductive coatings, the configuration of the electron column, the excitation voltage and choice of electron signal to use for imaging, and the pre- and post-processing of image data all influence the results of our analyses. The second challenge involves the processing of extremely large volumes of image data. Individual images can be larger than 100 gigabytes in size, too large for many of the existing tools for image analysis to open and segment. The third challenge, involves the upscaling of our observations from the nanometer scale of the pore space to the vertical thickness of the formation (hundreds of meters) and the basin scale (hundreds of kilometers). This paper presents an evaluation of how the details of sample preparation, image acquisition, and image processing influence the results of porosity and Total Organic Carbon (TOC) analysis from SEM image data in shale reservoirs.

Analysis of shale reservoir images has focused primarily on the distribution and abundance of organic material and porosity, as these two parameters have been identified as having economic significance. Robust segmentation of porosity, however, has not proven to be simple. Results have been shown to be highly variable depending upon the quality of the image, the software tools used, the segmentation scheme employed, and the experiences and preferences of the person(s) performing the analysis. In order to illustrate this, we collected a series of secondary electron images at progressively lower accelerating voltages. In general, as accelerting voltage decreases, the segmented porosity increases. This same trend is observed in BSE image analysis, although porosity segmented from the same field of view collected using BSE is routinely lower than that segmented from the equivalent SE image. Preprocessing of images (applying a denoising filter, for example) may result in the elimination of very small pores together with noise. Image filtering is nearly always required when ion milling artifacts are present on the surface,however, due to common misidentification of topographic lows as porous regions during segmentation. If pre-segmentation filtering is not performed, post processing to remove noise artifacts (by applying a minimum size filter, for example) can reduce the number of segmented pores by several thousand but reduce the percentage of segmented porosity only negligibly (hundredths of a percent). Pore size distributions from these two approaches, however, will be vastly different. We will show the results of a comparative study of segmentation of a suite of images performed by different operators using different software packages, and identify the best imaging conditions and image processing approach so that all results are within error bars. 

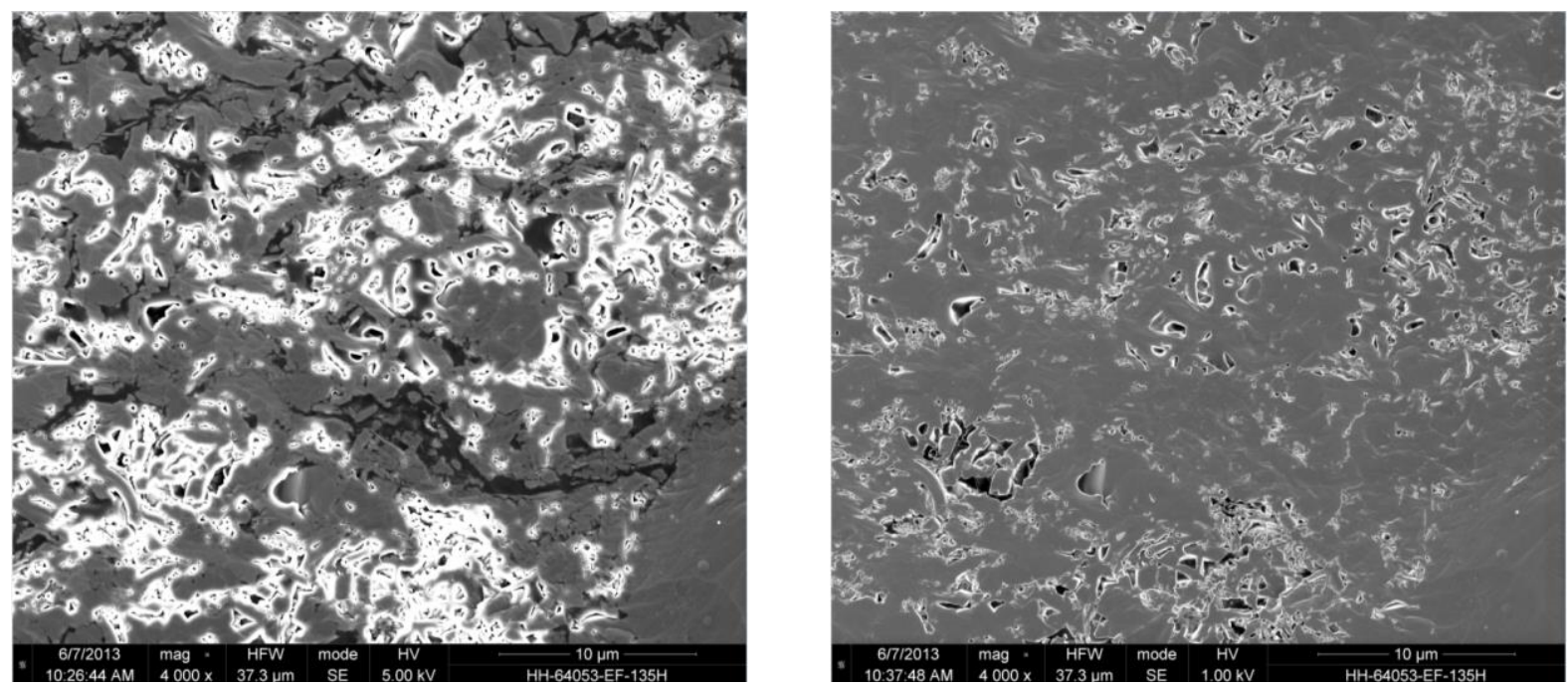

Figure 1. Secondary electron images of pore space in a shale reservoir. Accelerating voltage is $5 \mathrm{kV}$ for image at left, and $1 \mathrm{kV}$ for image at right. Bright rims around pores highlight their location but make segmentation of porosity more difficult. Note that organic material is readily identfied at $5 \mathrm{kV}$ but not at $1 \mathrm{kV}$ (red arrows). Segmented porosity for image A is $0.3 \%$. Segmented porosity for image B is $2.4 \%$.
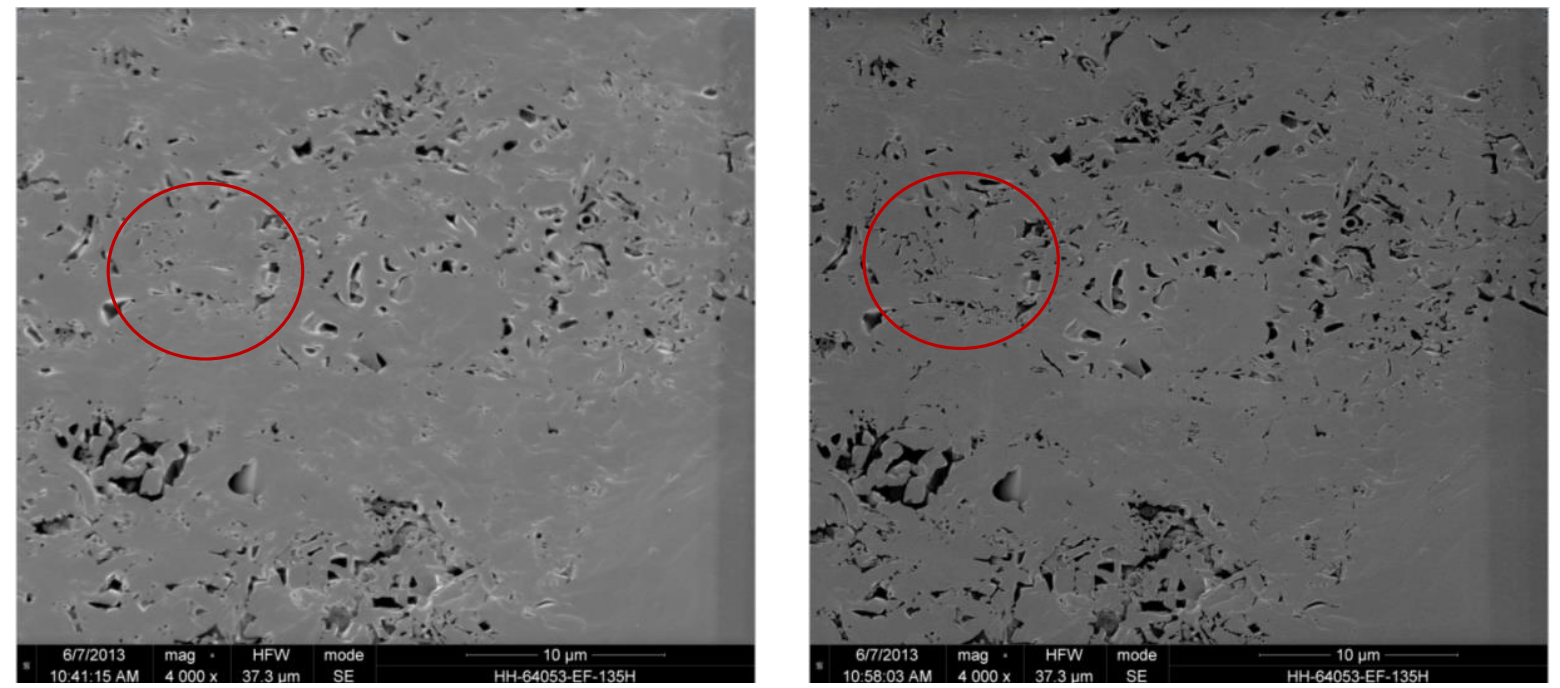

Figure 2. Secondary electron images of pore space in a shale reservoir. Accelerating voltage is $750 \mathrm{~V}$ for image at left, and $350 \mathrm{~V}$ for image image at right. Note the increase in the number of small pores in the region highlighted by the red circle. Segmented porosity for image A is $4.75 \%$. Segmented porosity for image $\mathrm{B}$ is $6.3 \%$. 